\title{
Efficient Optimization of An Individual Tree Growth Model*
}

\author{
Atsushi Yoshimoto ${ }^{1}$, Gonzalo L. Paredes V., ${ }^{1,2}$ J. Douglas Brodie ${ }^{1}$
}

\begin{abstract}
The PATH algorithm (Paredes and Brodie,1987) is interpreted by means of the calculus of variations. Using the PATH algorithm, a new dynamic programming model called Stand Optimization System (SOS) is developed. The system is incorporated into a growth simulator constructed by Arney (1985). Further limitation of optimality on the PATH algorithm and the relationship between the Lagrange multiplier and the decision variable are discussed.
\end{abstract}

Keywords: forest stand level optimization, optimal thinning regime, dynamic programming, calculus of variation

\section{Introduction}

Determination of the optimal thinning regime and rotation age has been a main problem in even-aged forest stand management. The widespread application of operations research techniques has been contributing to solving the stand level optimization problem. The dynamic programming approach has been developed and extensively applied in recent years.

The dynamic programming approach was applied to the forestry field by Amidon and Akin (1968), Arimizu (1959), and Schreuder (1969). Early scientists used a two-descriptor, i.e., volume and age, dynamic programming model in order to cluster each state (Brodie et al., 1978; Chen et al.,1980; Kilkki and Väisänen, 1970). Brodie and Kao (1979) proposed a three descriptor dynamic programming model (number of trees, basal area, and age), using an existing stand growth simulator for Douglas-fir (Pseudotsuga menziesii [Mirb.] Franco) called DFIT.

This three-descriptor dynamic programming model with forward recursive approach has been extended and adapted for other decision variables and species. Riitters et al. (1982) presented a dynamic programming model with timber production and grazing control joint optimization using a ponderosa pine (Pinus ponderosa Dougl. ex Laws.) growth model called PPINE. Haight et al. (1985) proposed a dynamic programming model with thinning and rotation age optimization using lodgepole pine (Pinus contorta Doug. ex Loud.). A hardwood release and thinning optimization dynamic programming model was constructed for loblolly pine (Pinus taeda L.) by Valsta (1985). Torres (1987) proposed a thinning optimization dynamic programming model for a Pinus hartwegii growth model. In comparison with the above three or more descriptor models, a dynamic programming model optimizing both thinning and rotation age for an individual-tree red pine model (Pinus resinosa Ait.) was completed by Martin and Ek (1981) with two descriptors.

Although some of the above models reduced calculation effort to select the optimal activity by using the "neighborhood storage location" technique (Brodie and Kao, 1979), dynamic programming still has computational limitations. The more complicated the growth simulator, the more memory and calculations are required (Hann and Brodie, 1980).

Paredes and Brodie (1987) resolved the memory and calculation problem by utilizing both network theory and the theory of the Lagrange multipliers. Their efficient way of selecting the optimal path reduced the number of calculations and associated computer storage in comparison with the traditional dynamic programming approach. Since the number of elementary calculations in traditional dynamic programming increases exponentially with problem size, the efficiency is greater with larger problems. However, searching for the optimal value of the Lagrange multiplier remains incomplete. Two other problems called trade-off problems were not dealt with by Paredes and Brodie (1987), one of which deals with situations where no thinning is applied at a certain stage, and the other of which deals with problems associated with insufficient look-ahead period when intensive thinning is applied.

This paper describes and interprets their algorithm (called the PATH algorithm) from a different point of view, in which the technique does not use the Lagrange multiplier. Then a new dynamic programming model is proposed by using the PATH algorithm and a growth simulator called Stand

\footnotetext{
${ }^{1}$ Oregon State University, USA, ${ }^{2}$ Universidad Austral de Chile, Chile

* This is a reproduction from its original publication of Yoshimoto et al. (1988) USDA Forest Service General Technical Report RM-161:154-162 with minor correction.
} 
Projection System (SPS) (Arney, 1985). The trade-off problems are also solved by a new algorithm called Multi-Stage PATH (MSPATH).

\section{Optimizing Formulation}

\subsection{The PATH Algorithm by the Lagrange Multiplier Approach}

Paredes and Brodie (1987) proposed the optimization problem with the Lagrange multiplier as follows:

$$
\max f_{N}\left(Y_{N}\right)=\sum_{n=1}^{N} A_{n}\left(T_{n}\right)+\sum_{n=1}^{N} \mu_{n}\left[X_{n}-T_{n}+G_{n+1}\left(Y_{n}\right)\right]
$$

where $A_{n}$ is the return from thinning, $Y_{n}$ is the stand volume after thinning, $X_{n}$ is the stand volume before thinning at stage $n, G_{n+1}$ is growth from stage $n$ to stage $n+1$ based on $Y_{n}$ and $\mu_{n}$ is the Lagrange multiplier at stage $n$. They modified the formulation in the dynamic programming problem as:

$$
f_{n}\left(Y_{n}\right)=\max _{\left[T_{n}\right]}\left[A_{n}\left(T_{n}\right)+\mu_{n}\left[X_{n}-T_{n}+G_{n+1}\left(Y_{n}\right)\right]\right]+f_{n-1}\left(Y_{n-1}\right)
$$

The PATH algorithm does not utilize the state variables of the growth model for storing the optimal residual stand at each state with a fixed Lagrange multiplier, so that computational task was vastly diminished.

Searching for the optimal level of $\left[T_{n}, \mu_{n}\right]$, this objective function can be optimized. If the dimensions of both the first term and the second term on the right-hand side of Eq. [2] are monetary units, the Lagrange multiplier, $\mu$, can be interpreted as the price per unit of resource including its growth potential. In physical objective examples, the Lagrange multiplier can be estimated as simply 1 . The control decision concerns both the direct return from the thinning and the return from the future stand. Although it is possible to guess the Lagrange multiplier, it is not always guaranteed that such a value is optimal. Then it is necessary to search for the optimal Lagrange multiplier. The difficulty of searching for the optimal Lagrange multiplier $\mu_{n}$, can be eliminated by the following procedure.

\subsection{The PATH Algorithm by the Calculus of Variations}

The total return $V\left(t_{n}\right)$ at stage $n$ (time $t_{n}$ ) is the summation of marginal return over time:

$$
\begin{aligned}
V\left(t_{n}\right) & =\int_{t_{0}}^{t_{n}} M(t) d t \quad \text { in the continuous case, } \\
& =\sum_{i=0}^{n} M\left(t_{i}\right) \quad \text { in the discrete case. }
\end{aligned}
$$

where $M(t)$ is the marginal return at time $t$. Once thinning, $T$, is implemented at stage $1\left(\right.$ time $\left.t_{1}\right)$, the objective function, $V\left(t_{n}\right)$ is divided into two parts as:

$$
V\left(t_{n}, T\right)=\int_{t_{0}}^{t_{1}} M(t, T) d t+\int_{t_{1}}^{t_{n}} M(t, T) d t
$$

The first integrand on the right-hand side is equal to the sum of thinning and residual stand after thinning at stage 1 . The second integrand represents the sum of growth after thinning from stage 1 to stage $n$ (time $t_{n}$ ). Therefore, $V\left(t_{n}, T\right)$ represents the sum of returns from both thinning at stage 1 and the future stand at stage $n$. If the optimal thinning regime is required, the objective function becomes:

$$
V^{*}\left(t_{n}, T\right)=\max _{0<[T] \leq V\left(t_{1}\right)} V\left(t_{n}, T\right)=\max \left\{\int_{t_{0}}^{t_{1}} M(t, T) d t+\int_{t_{1}}^{t_{n}} M(t, T) d t\right\}
$$


It is obvious that the first integrand on the right hand side is constant because the current thinning cannot affect the previous stand. Then Eq.[5] becomes:

$$
V^{*}\left(t_{n}, T\right)=\max _{[T]} V\left(t_{n}, T\right)=V\left(t_{1}\right)+\max _{[T]} \int_{t_{1}}^{t_{n}} M(t, T) d t
$$

This approach to interpretation of the PATH algorithm is one of the classical calculus of variations problems (Intrilligator, 1971). The classical calculus of variations problem is to find the arc lying in a given plane and connecting two specified points in the plane by an arc of shortest length (Dreyfus, 1965). Thus, in this context, the classical calculus of variations problem can be interpreted as that of choosing the optimal thinning strategy, which satisfies the boundary condition and maximizes the integral or summation objective functional, $V(\cdot)$.

Schreuder (1971) specified a problem of the optimal strategy for an even-aged forest in the calculus of variations. Although specifying a continuous problem, Schreuder (1971) turned the calculus of variations problem into a dynamic programming problem because when the necessary conditions for an optimum (Dreyfus, 1965) were obtained for the general expression, a higher than first-order nonlinear differential equation, such as the Euler equation, resulted. Moreover, even if it could be solved, it would be necessary to investigate numerically all the roots to locate the global maximum because the conditions are only necessary and not sufficient. Then it was impossible for Schreuder (1971) to obtain a closed-form expression.

However, if we know exactly what the objective function looks like, then it is not necessary to solve the Euler equation to obtain a general solution. Furthermore, necessary conditions function as useful tests, which can eliminate candidate solutions, even if they are not sufficient to prove global optimality. After satisfying necessary conditions, it becomes obvious that there is an optimal trajectory. If we have such a trajectory that satisfies a given problem, that trajectory could be optimal.

Let the objective be the maximization of total volume, $J$, i.e.,

$$
\begin{aligned}
\max _{[X]} J & =\sum_{n=0}^{N} \int_{t_{n}}^{t_{n+1}} X_{n}^{\prime} d t, \\
X & =\left(X_{0}, X_{1}, X_{2}, \ldots, X_{N}\right), X_{N+1}\left(t_{N+1}\right)=0, X_{0}\left(t_{0}\right)=0
\end{aligned}
$$

where $X$ is a vector describing a thinning regime.

As mentioned by Everett (1963), if the value of $X_{i}$ is decided independently in each cell, the sum is obviously maximized by simply maximizing the following objective function with respect to $X_{n}$, so that we solve recursively:

$$
\max _{[X]} J_{n}=\max _{[X]} \int_{t_{n}}^{t_{n+1}} X_{n}^{\prime} d t
$$

In the PATH algorithm, it is assumed that the choice of $X_{i}$ does not affect the optimal path after the next stage, which is equivalent to the above assumption. The reason is that the stand, which provides the maximum sum of marginal return, or growth during the previous period, seems most likely to create the optimal stand at the next stage. However, there is a case where this assumption does not hold. Such a case is discussed later.

In a given problem, for any $X_{n}(t)$ satisfying boundary conditions, all necessary conditions provided by Dreyfus (1965) hold. Thus what should be done next is to search for the optimal trajectory among admissible ones. Let's define notation as follows:

$$
\begin{aligned}
Y_{n}= & \text { vector describing the stand at stage } n \text { before a decision } T_{n}, \\
X_{n}= & \text { vector describing the stand at stage } n \text { after a decision } T_{n}, \\
T_{n}= & \text { vector describing the decision variable (thinning) at stage } n, \\
& \quad \text { transferring the stand } X_{n} \text { into } Y_{n}, \\
X_{n}^{\prime}= & \text { stand growth at range }\left(t_{n}, t_{n+1}\right) .
\end{aligned}
$$

Therefore, among these variables, some relationships are formulated:

$$
X_{n}+T_{n}=Y_{n}
$$




$$
X_{n}+\int_{t_{n}}^{t_{n+1}} X_{n}^{\prime} d t=Y_{n+1}
$$

From these equations, the objective function [8] can be converted into the following function:

$$
\begin{aligned}
\max _{\left[X_{n}\right]} J_{n} & =\max _{\left[X_{n}\right]} \int_{t_{n}}^{t_{n+1}} X_{n}^{\prime} d t \\
& =\max _{\left[X_{n}\right]}\left\{Y_{n+1}-X_{n}\right\} \\
& =\max _{\left[T_{n}\right]}\left\{Y_{n+1}-Y_{n}+T_{n}\right\}
\end{aligned}
$$

Since $Y_{n}$ is constant for all admissible trajectories [the principle of optimality, Dreyfus (1965)], $Y_{n}$ can be eliminated from the objective function, resulting in the new objective function:

$$
\max _{0<T_{n}<Y_{n}} J_{n}=Y_{n+1}+T_{n}
$$

As a result, a sequence of $T_{i}^{*}$ which optimizes the objective function [12] in each cell, can constitute the optimal thinning regime maximizing the original objective function [7]. It is also possible to determine the optimal rotation age in terms of maximizing mean annual increment of $J$ with respect to $t_{n+1}$. In other words, setting up the maximum problem as:

$$
\max _{[N]} \max _{[T]} \frac{1}{t_{N+1}} \sum_{n=0}^{N} \int_{t_{n}}^{t_{n+1}} J\left(X_{n}, X_{n}^{\prime}, t\right) d t
$$

the optimal rotation age can be obtained at the same time. If the optimal stand at each stage is obtained, Eq.[13] becomes:

$$
\max _{[N]} \max _{[T]} \frac{1}{t_{N+1}} \sum_{n=0}^{N}\left(Y_{n+1}^{*}+T_{n}^{*}-Y_{n}^{*}\right)=\max _{[N]} \frac{1}{t_{N+1}}\left(T_{1}^{*}+T_{2}^{*}+\cdots+T_{N}^{*}+Y_{N+1}^{*}\right)
$$

where $Y_{N+1}^{*}$ and $T_{n}^{*}(n=1,2,, N)$ are the optimal stand volume at age $t_{N+1}$ and optimal thinning level at age $t_{n}$, respectively.

Given prices of inputs, outputs, and an appropriate discount rate, the production function can be converted into a net revenue equation where revenues and costs occurring at different points in time are properly discounted. Maximizing this expression yields the optimum patterns of inputs and outputs through time.

\section{Limitation of the Optimality}

In the PATH algorithm, it has been assumed that the optimal path at every stage is determined based on evaluations of the stand's reaction at the next stage. In other words, the return from the future stand is estimated on the basis of the next stage. Then the path provided is optimal as long as it is decided on this criterion, and also if necessary conditions for an optimum, from the calculus of variations problem, hold. However, two interesting situations can develop. The first situation occurs if there is no thinning at the next stage. The second situation occurs in conjunction with intensive thinning, when the look-ahead period is insufficient to evaluate impacts on the future stand. These situations are discussed below.

\subsection{The Impact on Optimality with No Thinning at the Next Stage and Intensive Thinning at the Current Stage}

Let's consider the first situation, at which it is assumed that $T_{1}$ at stage 1 is the optimal thinning level on the basis of stage 2 , and that there is no thinning at stage 2 based on stage 3 . Thus, if sufficient evaluation of the future stand based on stage 3 is done at stage 1 , there can be another optimal thinning level, $T_{1}^{\prime}$. If $T_{1}^{\prime}$ produces more objective value than the path provided by the PATH algorithm, the trade-off has to be evaluated at stage 3 . 
As for the second situation, it is possible for a stand with few trees to create a great potential growth over the long-term in a complicated stand growth simulator. This discrepancy also violates the assumption of the PATH algorithm that one-stage look-ahead period is sufficient to evaluate impact of the future stand. Then sufficient evaluation of the future stand should be implemented especially for a complicated growth simulator. These two situations suggested the following new PATH algorithm called Multi-Stage PATH (MSPATH) algorithm.

\subsection{Multi-Stage PATH (MSPATH) Algorithm}

The above two situations occur when the look-ahead period is insufficient to evaluate impact on the future stand. The MSPATH algorithm uses each possible look-ahead period at each stage in order to search for the optimal objective value at each future stage based on the different combinations of look-ahead period. In other words, the MSPATH algorithm searches for the optimal combination of look-ahead period from the initial stage to the final stage at the same time when the optimal thinning level is decided. Then MSPATH can decide where one-stage look-ahead is used, two-stage look-ahead is used and so on, and how much the optimal thinning level is for the optimal combination of look-ahead period. Then the optimal thinning regime at each stage can be obtained based on multi-stage look-ahead period. Figure 1 shows the possible combinations of look-ahead period by the MSPATH algorithm.

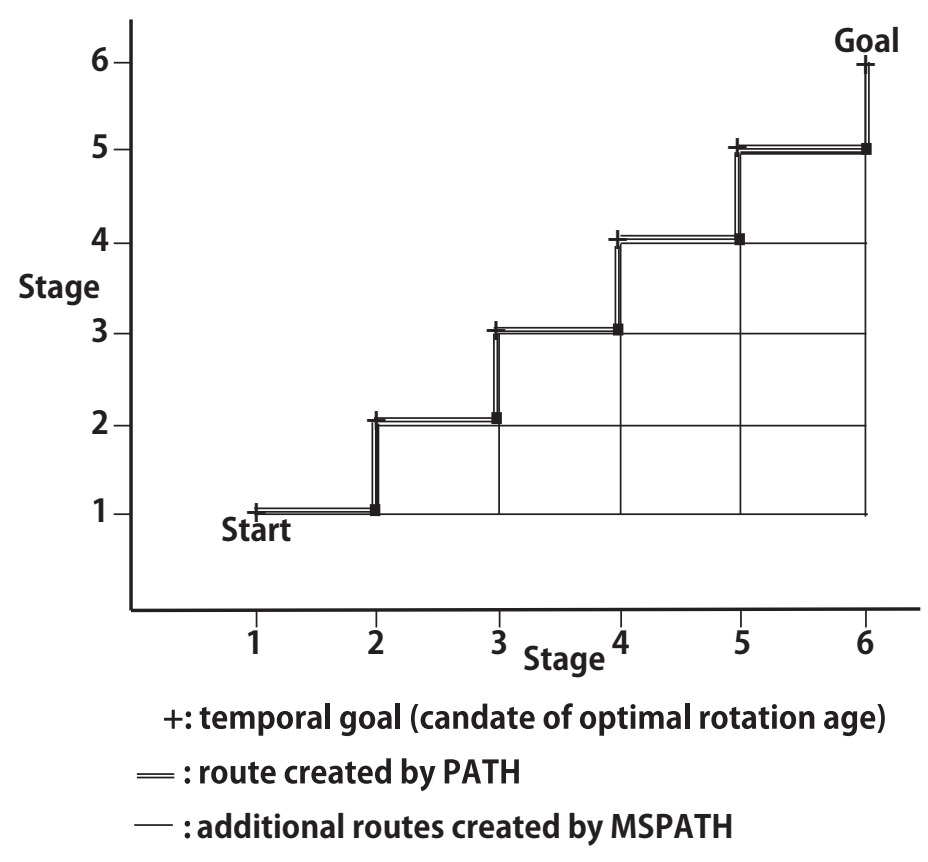

Figure 1. Possible routes by MSPATH

From the viewpoint of computational burden, MSPATH creates more computation than PATH. However, if the traditional dynamic programming algorithm is used in order to solve the same optimization problem as MSPATH by using SPS, the problem of insufficient look-ahead period also appears. That is, with the traditional dynamic programming algorithm, a one-stage look-ahead period, is utilized. Therefore, if a more accurate solution is needed for the traditional dynamic programming algorithm, the same technique as MSPATH utilizes to extend look-ahead period should be implemented. As a result, even if MSPATH creates more computational burden than PATH, it is still efficient in comparison with the traditional dynamic programming algorithm, and provides the optimal solution so long as necessary conditions for a calculus of variations problem hold. 


\section{Stand Optimization System (SOS)}

By using the SPS growth simulator (Arney, 1985), the Stand Optimization System models (SOS) are proposed with either the PATH algorithm or the MSPATH algorithm. The acronym SOS is used to distinguish the proposed optimization framework from the SPS simulation framework.

The SOS system developed here is classified as a deterministic, single descriptor, discrete-state, discrete-stage dynamic programming model. The problem solved utilizes a forward recursion. While searching for the optimal thinning level at each stage, it is possible for the user to use one of two different criteria in order to select the optimal thinning level.

One of them is such that once the objective value declines, the previous thinning level is labelled optimal. The other is such that after calculating all admissible solutions, the optimal thinning is selected among them. The following procedure is imbedded into the SOS model with the PATH algorithm. Since the basic procedure for the SOS model with the MSPATH algorithm is the same as the SOS model with PATH, optimization procedure discussion is excluded for the MSPATH algorithm here.

\subsection{Optimization Procedure}

Employing a forward recursion to find the optimal thinning regime and rotation age, SOS searches for the optimal thinning regime at each stage in the following way:

First SOS creates the initial forest stand structure having diameter distribution with individual tree height data and crown ratio data, which result from a yield table given by the user. After thinning an amount of trees, which is decided by the number of iterations calculated, and the interval of node given by the user, i.e., $N \times$ Interval, a residual stand grows until the next stage. At this stage, the sum of returns from both thinning and the future stand is compared with the previous one to store the best thinning level so far. If the user selects the first option, i.e., the unimodality assumption, once the objective function declines, SOS quits the iteration at this stage, and decides the previous thinning level is optimal. Otherwise, the iteration is continued until the number of the residual trees is less than the interval of thinning, then the best thinning level among admissible strategies is selected. SOS can also select the optimal thinning method at each stage among thinning from below, thinning from above, and thinning to a cut/residual ratio fixed as 1 .

If the thinning method "joint optimization" is selected by the user, after storing the best thinning level for one method at each stage, SOS does the same operation for two other methods so as to search for the best thinning level with each method at each stage. Comparing these three best objective values at each stage provides the optimal thinning method and level at each stage.

After determining the optimal trajectory at this stage, SOS sets up the initial forest structure at the next stage. Iterations continue over state and over stage until the last activity completes searching for the optimal trajectory from the initial stage to last stage. At the last activity, SOS searches for the optimal thinning level and rotation age at the same time by means of increasing rotation age by a 10-year step. In other words, first set rotation age 10 years after last activity, store the best objective value and thinning level. Then add 10 years to the previous rotation age, search for the optimal objective value, then compare the present best value to the previous one. If the previous one is greater than the present one, the previous age is regarded as the optimal rotation age after all activities. However, it is possible for the early stage to have more objective value than the rotation age selected by the above procedure. That is, the above procedure provides the optimal rotation age if the optimal rotation age is later than the last thinning time given by the user. Then SOS searches for the new optimal rotation age again from the initial stage to the rotation age calculated by the above procedure. As a result, optimization of both thinning regime and rotation age is completed. This procedure is summarized in Figure 2.

\subsection{Features of the SOS Model}

The objective function of SOS can be based on mean annual increment of basal area, total cubic feet, merchantable cubic feet or board feet as well as present net worth (PNW), and soil expectation value (SEV).

Combining thinning basis, such as trees per acre (TPA) or crown competition factor $(\mathrm{CCF})$, and thinning method, such as thinning from below, thinning from above, or thinning to a $\mathrm{c} / \mathrm{r}$ ratio of 1 , 


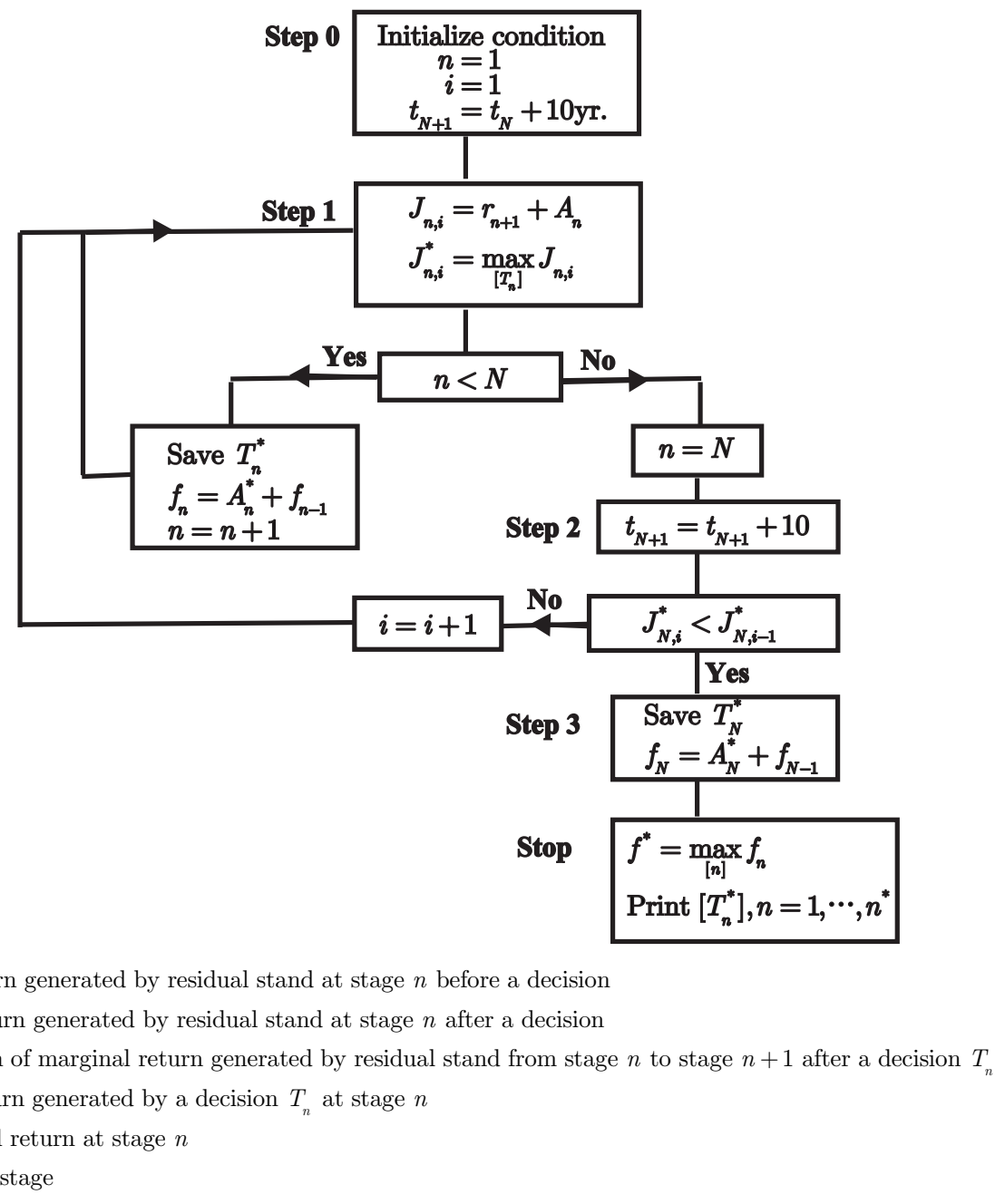

Figure 2. Flowchart of the PATH algorithm

can provide $2 \times 3=6$ possible thinning regimes. If thinning method joint optimization is selected, SOS indicates not only the optimal thinning level but also the optimal thinning method at each stage on either TPA or CCF basis.

For the sake of making the model simple, cost and revenue from thinning and final harvest are based on entry cost, stumpage price premiums, and other constant silvicultural costs. Entry cost is fixed over time for thinning and final harvest. Price equation per cubic foot is expressed as a function of DBH, which can be created by using either one linear equation or an equation with several continuous linear segments. It is also possible to utilize a different price equation for different species. These financial data, with interest rate, are utilized for economic optimization and in the financial reports of physical optimization. Although it is possible to use more complicated economic data, such as logging cost identical for each thinning level, cost is limited as above.

Since a price equation transforms a searching surface which is directly derived from physical data into a new surface which has economic information, it is necessary to take into account what kind of price equation can be used. In other words, even if the searching surface obtained from direct physical data is nicely concave, it is possible for it to become an irregular surface when the price equation is included. In such a case, one of the necessary conditions, the Weierstrass condition, which shows the condition for concavity over the control variable (thinning level), would be violated, as well as the Weierstrass-Erdman comer condition. For example, if a step-wise price equation is used, these conditions are not satisfied. This violation is shown by the relationship between the objective function and the decision variable, which would not be concave. Once these conditions are violated, the solution provided by SOS becomes the better solution, and not the best one. 


\section{Analysis with the SOS Model}

\subsection{Illustrative Example}

To demonstrate SOS with both the PATH and MSPATH algorithms, an input file and financial data are required. Characteristics of the data are:

species : Douglas-fir and western hemlock,

site index : 82 of Douglas-fir at 50-year breast-height-age basis,

region : Pacific Northwest Region.

Thinning is implemented at age 20,30, and 40 years. The basis of thinning is number of trees per acre, and the type of thinning is thinning from below with maximum DBH limit 100 inches (this value should be large so that thinning from below can be implemented at every diameter class).

In addition to this data, tree height, number of trees per acre, breast height age, and percent of crown ratio at each DBH class as well are given in Table 1.

Table 1. An Illustrative Example (Arney, 1985)

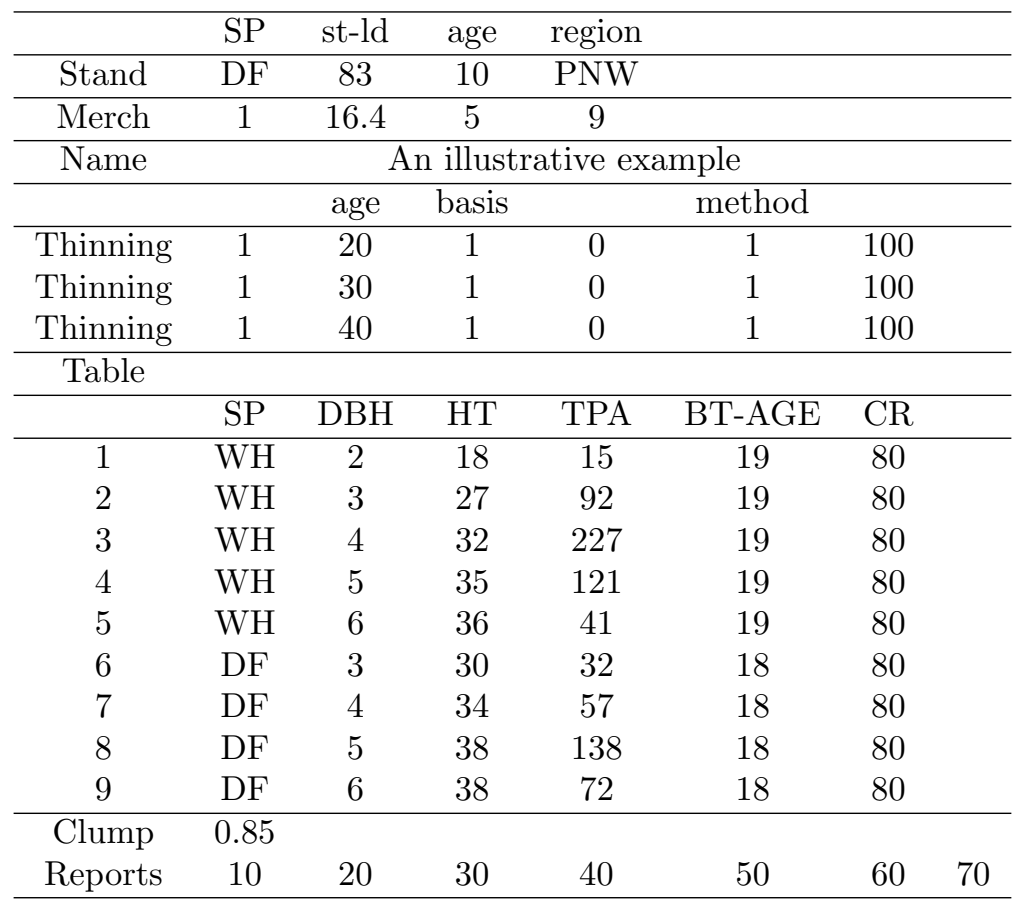

Also optimization basis, interval of node, and financial data are provided as follows:

$\begin{array}{ll}\text { optimization basis } & : \text { soil expectation value(SEV), } \\ \text { interval of node } & : 20 \text { trees per acre, } \\ \text { interest rate } & : 4 \% \\ \text { entry cost } & : \$ 50.00, \\ \text { coefficient for reducing thinning value } & : 0.80, \\ \text { regeneration cost } & : \$ 200 . / \text { acre at age } 0, \\ \text { price } / 1,000 \mathrm{ft}^{3} & : 200 \times \mathrm{DBH}+80 .\end{array}$

As shown in Table 2, if all activities are required, the optimal thinning regime and rotation age from the PATH algorithm are: 
thinning 180 trees per acre at age 20 , thinning 160 trees per acre at age 30, thinning 60 trees per acre at age 40 , clearcut at age 50 .

Table 2. Optimal thinning regime

\begin{tabular}{|c|c|c|c|c|c|c|c|c|c|c|}
\hline \multirow[b]{3}{*}{$\mathrm{AGE}$} & \multirow{2}{*}{\multicolumn{2}{|c|}{20 years }} & \multirow{2}{*}{\multicolumn{4}{|c|}{$\begin{array}{c}\text { Temporary rotation age } \\
40 \text { years }\end{array}$}} & \multirow{2}{*}{\multicolumn{2}{|c|}{50 years }} & \multirow{2}{*}{\multicolumn{2}{|c|}{60 years }} \\
\hline & & & & & & & & & & \\
\hline & PATH & MSPATH & PATH & MSPATH & PATH & MSPATH & PATH & MSPATH & PATH & MSPATH \\
\hline $20^{*}$ & $702^{* *}$ & 702 & 180 & 180 & 180 & 280 & 180 & 360 & 180 & 280 \\
\hline 30 & & & 522 & 522 & 160 & - & 160 & - & 160 & - \\
\hline 40 & & & & & 362 & 422 & 60 & - & 60 & 340 \\
\hline 50 & & & & & & & 302 & 342 & 200 & - \\
\hline 60 & & & & & & & & & 102 & 79 \\
\hline SEV & 3268 & 3268 & 3644 & 3644 & 3457 & 3555 & 3108 & 3179 & 2762 & 2799 \\
\hline
\end{tabular}

However, the results from the MSPATH have different thinning levels at each stage depending upon the temporal rotation age, which is characterized by MSPATH. Figure 3 shows the optimal path from the PATH algorithm in terms of stand volume and Figure 4 shows the path from the MSPATH algorithm. Figure 5 shows the searching surfaces over time, which can reveal the actual optimal rotation age. According to Figure 5, 30 years is the optimal rotation age for both models, which can differ depending on the economic data.

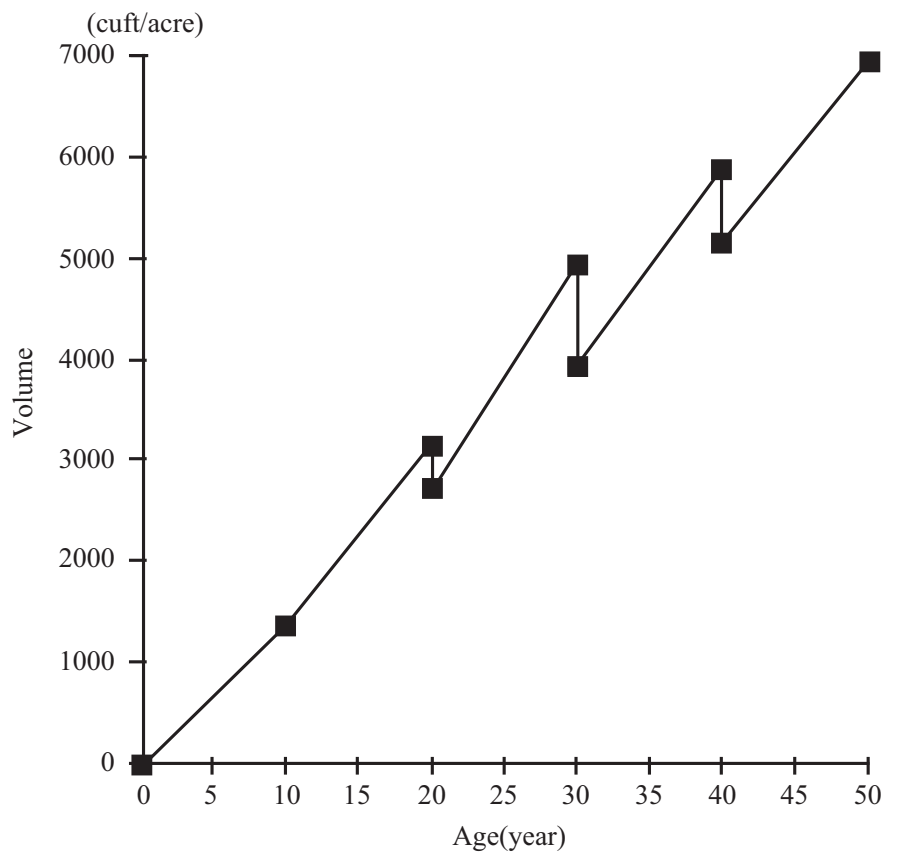

Figure 3. Optimal thinning regime by the PATH algorithm.

As expected, however, the difference of the objective value between the PATH algorithm and the MSPATH algorithm is less than $2 \%$, so if the user does not care about this small difference, the PATH algorithm can be recommended to save computational time.

\subsection{Relationship Between the Lagrange Multiplier and Optimal Thinning Regime}

As mentioned before, the PATH algorithm can be expressed in terms of the Lagrange multiplier as:

$$
f_{n}\left(Y_{n}\right)=\max _{\left[T_{n}\right]}\left[A_{n}\left(T_{n}\right)+\mu_{n}\left[X_{n}-T_{n}+G_{n+1}\left(Y_{n}\right)\right]\right]+f_{n-1}\left(Y_{n-1}\right)
$$




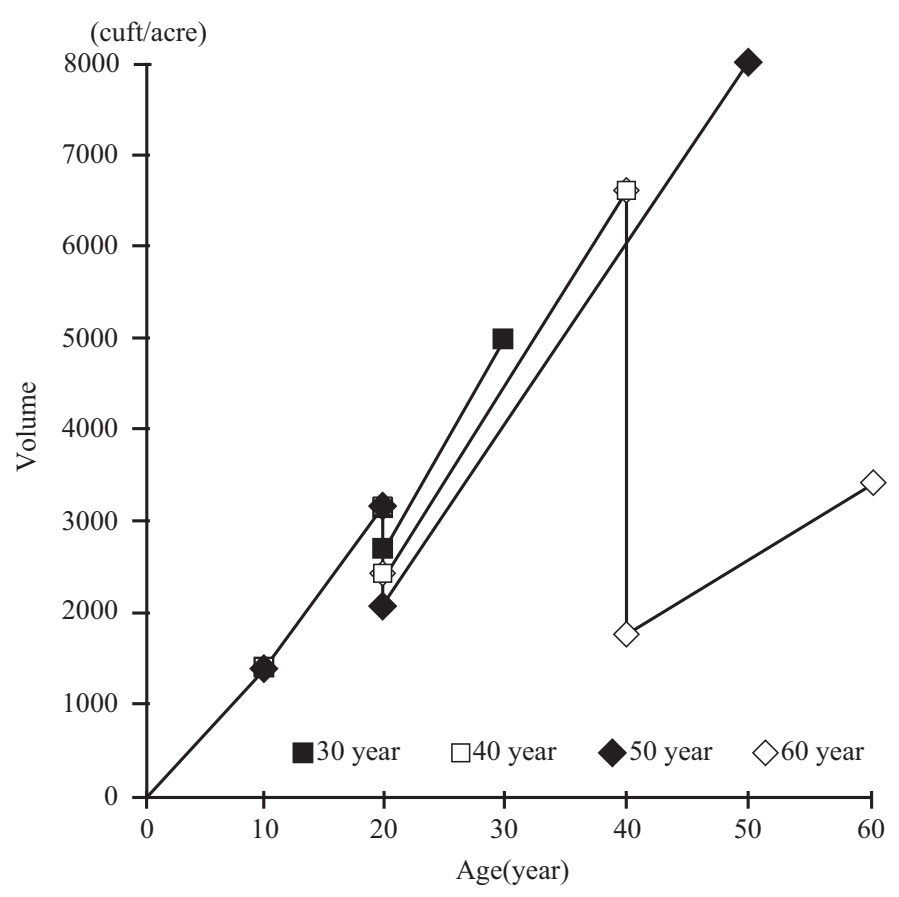

Figure 4. Optimal thinning regime by the MSPATH algorithm.

at the $n$-th stage. The PATH algorithm is also interpreted from a different point of view as:

$$
J_{n}=\max _{\left[T_{n}\right]}\left[A_{n}\left(T_{n}\right)+r_{n+1}\left(Y_{n+1}\right)\right]
$$

Since this objective function does not have the Lagrange multiplier, it is possible to estimate the Lagrange multiplier. Suppose that necessary conditions for a calculus of variations problem hold and sufficient look-ahead period is used. Then if the optimal solution is obtained by the above two functions respectively, the optimal thinning levels obtained by these two methods should coincide as long as the same interval of node is used. Then at the optimal point the following equation is satisfied:

$$
A_{n}^{*}\left(T_{n}\right)+\mu_{n}^{*}\left[X_{n}-T_{n}+G_{n+1}\left(Y_{n}\right)\right]=A_{n}^{*}\left(T_{n}\right)+r_{n+1}^{*}\left(Y_{n+1}\right)
$$

Solving for the Lagrange multiplier $\mu_{n}^{*}$, we can obtain:

$$
\mu_{n}^{*}=\frac{r_{n+1}^{*}\left(Y_{n+1}\right)}{X_{n}-T_{n}+G_{n+1}\left(Y_{n}\right)}=\frac{r_{n+1}^{*}\left(Y_{n+1}\right)}{Y_{n+1}}
$$

Therefore at the optimal point of the $n$-th stage, the Lagrange multiplier can be interpreted as the average return per unit volume at the $(n+1)$-th stage. If the basis of optimization is cubic-foot volume, the Lagrange multiplier becomes equal to 1 as was expected by Paredes and Brodie (1987).

Economically speaking, both the Lagrange multiplier and the decision variable correspond with each other. In other words, if the Lagrange multiplier is given, then the decision variable is determined at the optimal point. Then searching for the optimal allocation is limited to the range at which this relationship holds.

Figure 6 shows the relationship between the Lagrange multiplier derived from Eq.[17] and the decision variable, thinning level. The higher thinning level, the larger the Lagrange multiplier. Also the greater the stage, the less the Lagrange multiplier.

The Lagrange multiplier derived is interpreted economically not only as the shadow price or the opportunity cost but also as the marginal value per unit volume of resource at each stage by which the maximum attainable value of resource could be increased if an additional unit of resource were to become available (Dorfman 1961, Paredes and Brodie, 1987). According to Eq.[17], the Lagrange 


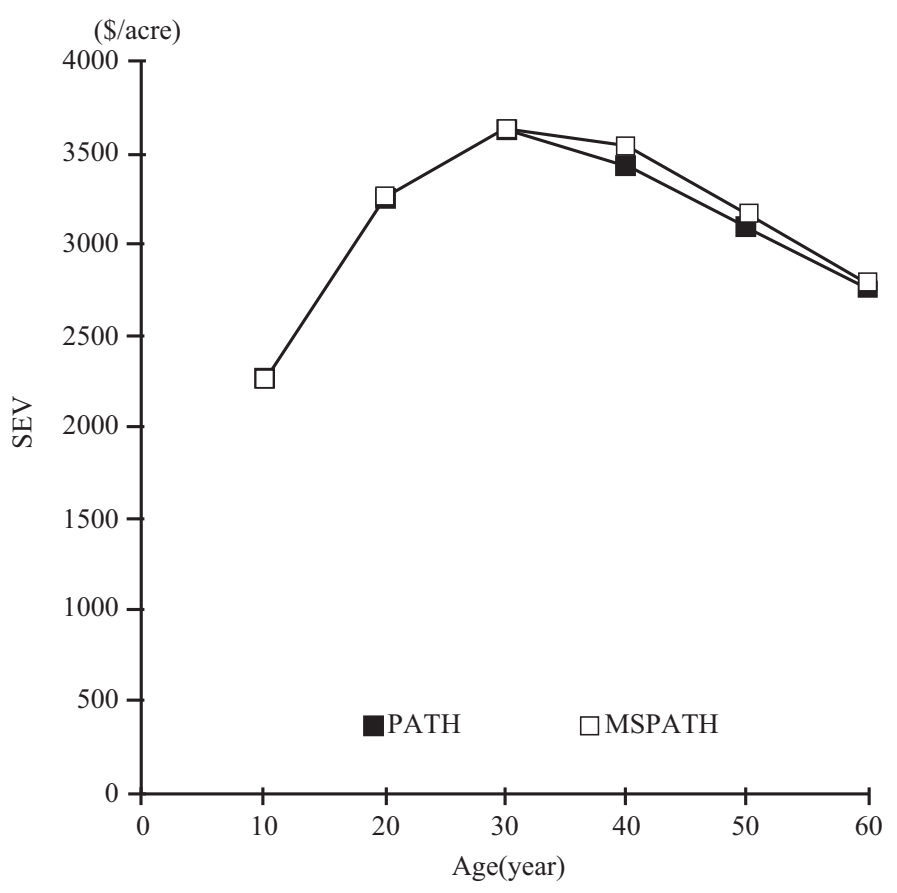

Figure 5. Searching surface of SEV.

multiplier having the above interpretation at each stage should be estimated based on the future stand, not the current.

\section{Conclusions}

The objective of this paper was to develop a new dynamic programming model using the SPS growth simulator (Arney,1985), employing the PATH algorithm (Paredes and Brodie,1987). This model called SOS can optimize both thinning regime and rotation age based on either mean annual increment of the given physical basis, present net worth, or soil expectation value, as long as necessary conditions for a calculus of variations problem hold and one-stage look-ahead period is sufficient to evaluate the future stand. Once either one of these conditions is violated, or one-stage look-ahead period is insufficient, the solution obtained by PATH becomes the better solution, and not the best solution. Then SOS based on the MSPATH algorithm is also proposed in order to solve the optimization problem when one-stage look-ahead period becomes insufficient in evaluating the return from the future stand. However, once a necessary condition is violated, the solution provided by MSPATH becomes better, and not best.

The modification of the PATH algorithm by the calculus of variations in order not to use the Lagrange multiplier allows one to recognize the efficient PATH algorithm easily. Once the optimal resource allocation is obtained, the optimal value of the Lagrange multiplier can be calculated automatically as well, in terms of the relationship between the objective function with the Lagrange multiplier and without.

Directly treated as a decision variable, the unit thinning level given by the user determines the number of iterations at each stage, or the residual level before thinning divided by the unit thinning level. This technique eliminated so many calculations that the joint optimization of thinning methods and the optimization of rotation age are completed with less computation in one run of SOS than the traditional dynamic programming algorithm. Thus the calculation task and memory required to store optimal stands is vastly diminished to utilize very complex forest stand level production models.

Brodie and Haight (1985) indicate that when thinning from above is incorporated in an optimization model, where growth is driven by top-height a violation of the principle of optimality can occur unless state space is expanded to include top-height. Although SOS growth is driven by top- 


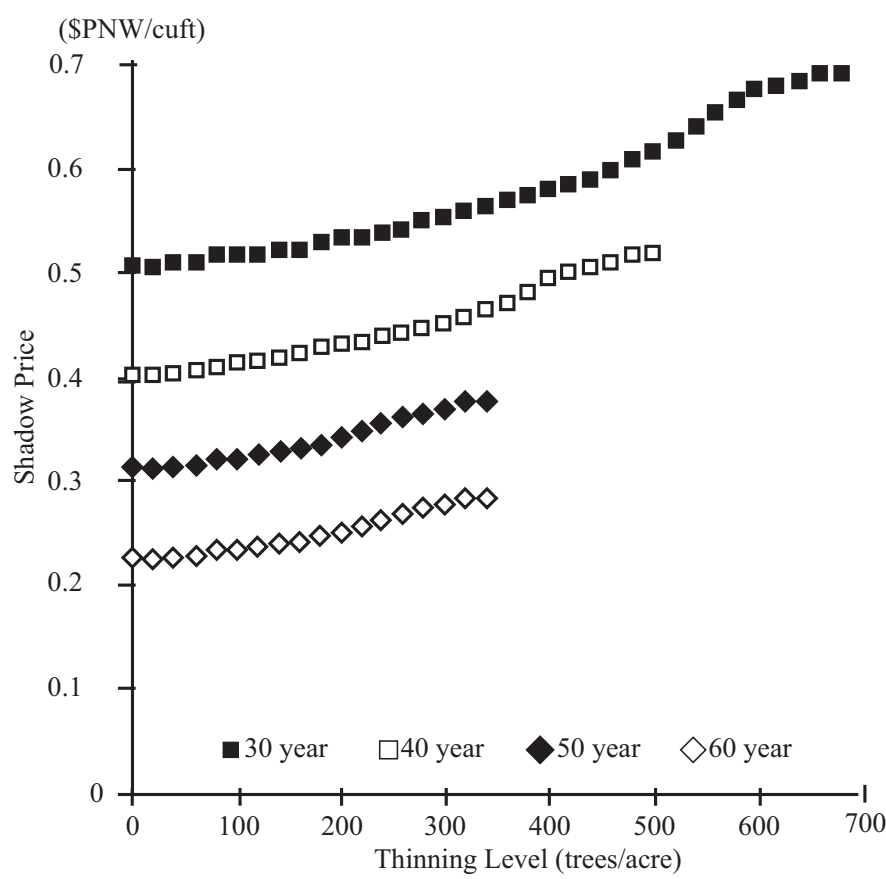

Figure 6. Relationship between shadow price (Lagrange multiplier) and thinning level

height, the implied expansion of state space becomes unnecessary through the process of evaluating the future stand (after thinning from above) as part of the objective function. The same problem of suboptimization occurs, however, if the future stand productivity is not projected sufficiently forward. In such case, the MSPATH algorithm can resolve it.

\section{References}

Amidon, E.L. and Akin, G.S. (1968) Dynamic programming to determine optimal levels of growing stock. For. Sci. 14: 287-291.

Arimizu, T. (1959) Dynamic programmings in forestry. J. Jpn. For. Soc. 41: 448-458.

Arney, J.D. (1985) A modeling strategy for the growth projection of managed stands. Can. J. For. Res. 15: 511-518.

Brodie, J.D., Adams, D.M. and Kao, C. (1978) Analysis of economic impacts on thinning and rotation for Douglas-fir using dynamic programming. For. Sci. 24: 513-22.

Brodie, J.D. and Haight, R.G. (1985) Optimization of silvicultural investment for several type of stand projection system. Can. J. For. Res. 15: 188-191.

Brodie, J.D. and Kao, C. (1979) Optimizing thinning in Douglas-fir with three-descriptor dynamic programming to account for accelerated diameter growth. For. Sci. 25: 665-672.

Chen, C.M., Rose, D.W. and Leary, R.A. (1980) Derivation of optimal stand density over time-a discrete stage, continuous state dynamic programming solution. For. Sci. 26: 217-227.

Dorfman, R. (1969) An economic interpretation of optimal control theory. Amer. Econ. Rev. 59: $817-831$.

Dreyfus, S.E. (1965) Dynamic programming and the calculus of variations. The Rand Corporation $511 \mathrm{p}$.

Everett, H. (1963) Generalized LaGrange multiplier method for solving problems of optimum allocation of resources. Oper. Res. 11: 399-417. 
Haight, R.G., Brodie, J.D. and Dahms, W.G. (1985) A dynamic programming algorithm for optimization of lodgepole pine management. For. Sci. 31: 321-330.

Hann,D.W. and Brodie, J.D. (1980) Even-aged management: basic managerial questions and available or potential techniques for answering them. USDA For. Serv. Gen. Tech. Rep. INT-83, $29 \mathrm{p}$.

Intrilligator, M.D. (1971) Mathematical optimization and economic theory. Englewood Cliffs, NJ, Prentice-Hall Inc. 597p.

Kilkki, P. and Väisänen, U. (1970) Determination of the optimum cutting policy for the forest stand by means of dynamic progmming. Acta For. Fenn. 102: 22p.

Martin, G.L. and Ek, A.R. (1981) A dynamic programming analysis of silvicultural alternatives for red pine plantations in Wisconsin. Can. J. For. Res. 11: 370-379.

Paredes V., G.L. and Brodie, J.D. (1987) Efficient specification and solution of the even-aged rotation and thinning problem. For. Sci. 33: 14-29.

Riitters, K., Brodie, J.D. and Hann, D.W. (1982) Dynamic programming for optimization of timber production and grazing in ponderosa pine. For. Sci. 28: 517-526.

Schreuder, G.F. (1968) Optimal forest investment decisions through dynamic programming. School of Forestry Bulletin, Yale University. 72p.

Schreuder, G.F. (1971) The simultaneous determination of optimal thinning schedule and rotation for an even-aged forest. For. Sci. 17: 333-339.

Torres R., J.M. (1987) Economic analysis of several alternatives of forest management for Pinus hartwegii. MS thesis, Oregon State Univ. 126p.

Valsta, L.T. (1985) An economic analysis of hardwood treatment in loblolly pine plantations: a whole rotation dynamic programming approach. MS thesis, Oregon State Univ. 69p. 